\title{
Initial Experiments toward Automated Robotic Implantation of Skew-Line Needle Arrangements for HDR Brachytherapy
}

\author{
Animesh Garg, Timmy Siauw, Dmitry Berenson, Adam Cunha, \\ I-Chow Hsu, Jean Pouliot, Dan Stoianovici, Ken Goldberg
}

\begin{abstract}
One of the goals of automation is to increase the reliability and quality of a given process. This study aims to automate high dose rate brachytherapy (HDR-BT), a radiation therapy that places radioactive sources at the site of the tumor using needles. Although HDR-BT has a high rate of clinical success in curing prostate cancer, it also has several side effects related to needle and dose trauma. A new planning algorithm from previous work optimizes needle arrangements using skewlines (non-parallel, non-intersecting lines). This paper presents initial experiments towards an automated system for implanting skew-line needle arrangements computed from a planning system. We describe the interface, calibration and integration of the robotic hardware with the planning system, and present experiments using our robotic system to implant needles into anatomically-correct tissue phantoms. Results suggest that this system can achieve HDR-BT treatment objectives with reduced trauma to organs and low demands on operator skill, thus making the procedure repeatable at lower cost. We believe that in the future, robotic HDR-BT will result in improved treatment quality with reduced dependence on physician skill.
\end{abstract}

\section{INTRODUCTION}

In the U.S. alone, over 40,000 cancer cases are treated with brachytherapy each year [1]. Brachytherapy places a radioactive source inside malignant tissue ("brachys": Greek for "proximal"), and it is an effective treatment for tumors in the prostate, cervix, breast, and other areas of the body [1]. In high dose rate brachytherapy (HDR-BT), the radiation is delivered directly to the tumor by a radioactive source which is sequentially threaded through an arrangement of temporarily inserted needles. Currently, the needle arrangement is implanted manually by physicians; outcomes and sideeffects vary with physician skill and experience. Recent studies confirm that existing procedures can result in acute side-effects such as edema in tissue, incontinence and impotence [2, 3, 4]. Most of these side-effects are a result of excessive radiation and needle penetration through sensitive structures (urethra, bladder, rectum, penile bulb, cavernous veins, and neurovascular bundles) $[5,6,7,4,8]$

In the clinical procedure for prostate HDR-BT, hollow needles are inserted one at a time into the prostate through the perineum (the region between the testicles and anus). The insertion is made manually by a physician under the guidance of a trans-rectal ultrasound probe which provides real time imaging. To help guide the needles to the proper place, the standard approach uses a rigid needle template, a

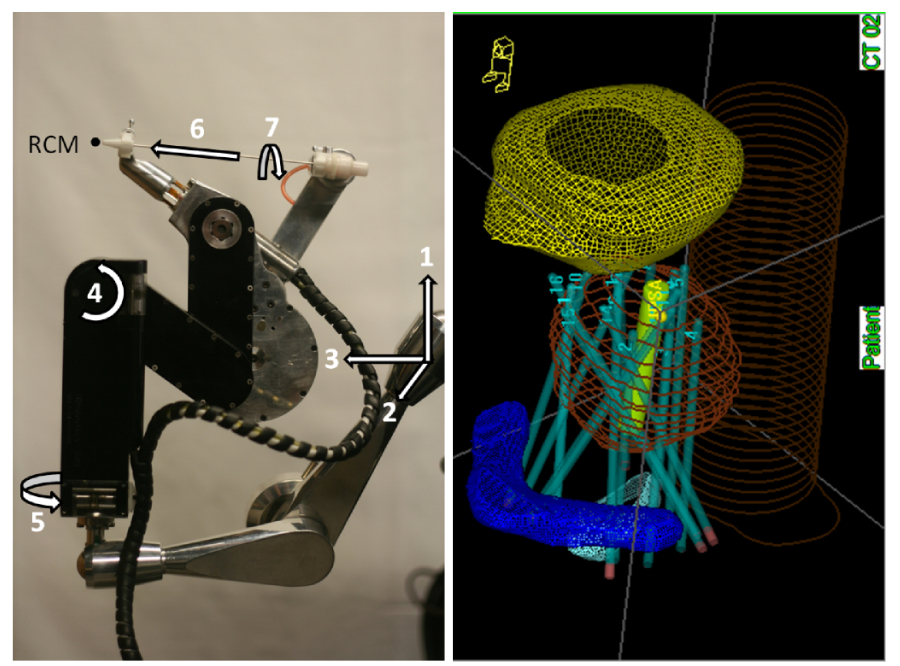

Fig. 1. The left figures shows 7-DoF Acubot-RND robot: a 3-DoF cartesian stage (1,2 and 3), a rotating center of motion (4 and 5), needle insertion and needle rotation (6 and 7). The right figure shows a skew-Line needle arrangement implanted by the semi-autonomous robot assisted system. The figure is obtained after CT-Scanning of tissue phantom, and contouring the structures in the digital form.

rigid device with parallel holes that helps control the needle trajectory during insertion. However, the template also restricts the possible insertion locations and directions. This often makes puncture of obstructions such as the bulb of the penis, and related vasculature, unavoidable. Recently a template-free, freehand approach was developed by physicians [9], however this approach requires a high degree of skill and clinical proficiency.

The primary goal of this study is to introduce principles of automation to HDR-BT. Specifically, we seek to improve treatment repeatability and reproducibility in a clinical environment. Reducing the reliance on the skill of the physician through automation will standardize treatment quality, increase adherence to treatment objectives, and reduced trauma to organs. In this study, we present initial experiments with a robotic HDR-BT system consisting of planning software, a needle insertion robot, Acubot-RND [10], and a human with no brachytherapy clinical experience. We show that, within our experimental setup, this system can consistently plan and implant needle configurations that avoid puncturing nonprostate organs and meet clinical radiation dose objectives for the procedure. We also show that the system does require 
excessive skill demands to operate. A photograph of the Acubot-RND and a needle configuration implanted by the robot is shown in Figure 1.

\section{BACKGROUND AND RELATED WORK}

Automation has been explored in a variety of medical applications: rehabilitation treatment in limbs[11, 12] and orthotic devices[13], drug delivery using MEMS devices[14],tumor detection using ultrasound[15], and clinical testing with "biorobots"[16]. In the present work, we apply principles of automation to brachytherapy.

There are two forms of brachytherapy: prostate permanentseed implant (PPI) and high dose rate (HDR) brachytherapy. In PPI-BT, needles are used to implant radioactive seeds which are left in the patient after the procedure. Usually only one needle is inserted into the body at any given time. The dose distribution is controlled through the configuration of seeds. In HDR-BT, a set of needles are inserted into the patient in a specific arrangement and a radioactive source is sequentially threaded through each needle using a device called a remote afterloader. The dose distribution is controlled by the dwell times spent at pre-specified positions in each needle.

The clinical HDR-BT workflow has five main steps: needle planning, needle insertion, anatomy segmentation, dose planning, and dose delivery. There has been studies in automating each of these steps. For example, there has been extensive effort in developing planning systems for computing optimal dose distributions for both PPI- and HDR-BT [17, 18, 19, 20, 21, 22]. These dose planning systems allow physicians to compute dose distributions without directly adjusting the dwell times to meet treatment objectives. Since the set of possible dose distributions depends on the implanted needle configuration, planning systems like Prostate Implant Planning Engine for Radiotherapy (PIPER) and Hybrid Inverse Planning and Optimization (HIPO) incorporate the positioning of needles into their dose planning model. However, these approaches were developed for the standard parallel-needle HDR template, which consists of a small set of needles that do not cover the set of needle configurations made possible with freehand techniques. There has also been work in autosegmentation of organ structures [23], and the afterloading device allows execution of HDR-BT dwell times without human intervention.

Needle insertion automation has been addressed through robotics. Custom robotic devices have been developed to address the clinical challenges of space constraints and safety requirements for needle insertion robots specially designed for prostate brachytherapy with trans-rectal ultrasound guidance $[24,25,26]$. Some of these brachytherapy-specific devices can insert needles at a continuum of locations and angles, emulating the degrees of freedom provided by freehand techniques, but can also place the tip of a needle with millimeter accuracy. However, the majority of current brachytherapy robot prototypes focus on PPI-BT [27, 28, 29, 30, 31], including the Acubot-RND [10, 32, 33], which is a needle insertion robot with applications to PPI-BT.

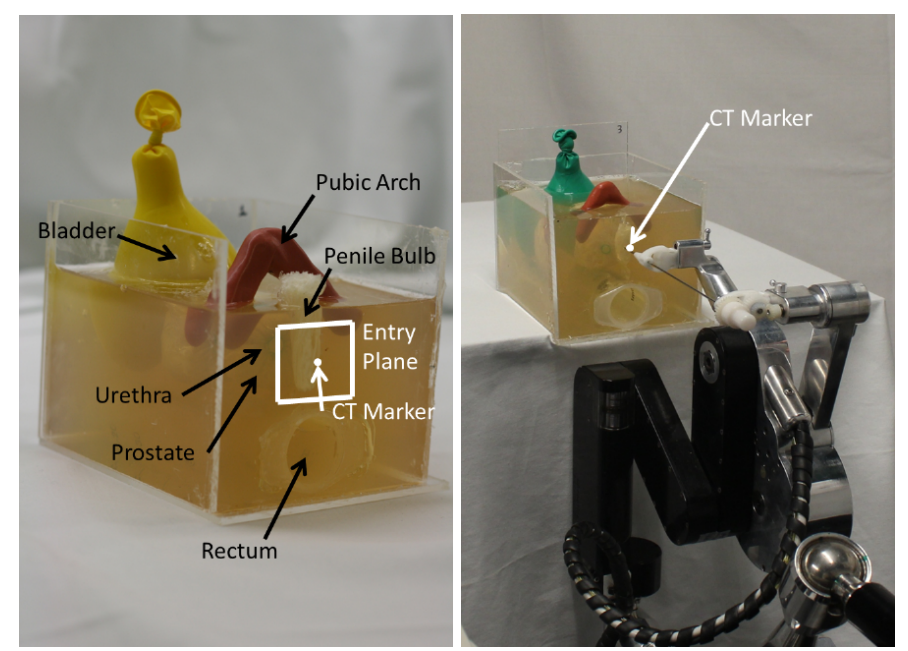

Fig. 2. Photograph of a prostate phantom used in this study (left) and insertion setup (right) for this experiment. The anatomy included was the prostate, urethra, bladder, penile bulb, pubic arch, rectum, and a CT marker for calibration with the robot and to represent the center of the perineum. The soft organs and connective tissues were gelatin-based. The pubic arch was constructed from clay putty. The Acubot-RND is registered to the CTmarker on the entry face of the phantom. In the initial state of robot: the needle is horizontal and orthogonal to entry plane with needle tip at the CT-marker.

The work presented in this study differs from previous work in several ways. Our system utilizes a novel needle planning system from previous work [34] which is designed to incorporate non-parallel, non-template needle geometries in planning. Thus, our needle planning system can take advantage of the degrees of freedom offered by robotic needle insertion devices. The Acubot-RND is designed for PPI-BT with needle insertion executed through manual joystick control. In this work, we augment Acubot-RND with custom software to allow coordinate inputs for automated insertion, and we design a workflow that allows Acubot-RND to perform HDR-BT. Our needle planning system is discussed in Section IV and the modifications to Acubot-RND are discussed in Section V. Combining automated planning and robotic execution, our work presents a system for performing HDR-BT with a higher degree of automation than previously possible.

\section{iII. Problem Statement}

In an environment consisting of a needle insertion robot, a CT-Scanner, planning software, and patient model, we will complete the following tasks: (1) compute a needle arrangement for the digitized anatomy of the patient that lies completely within the workspace of the robot, avoids nonprostate structures, uses needles economically, and meets dose objectives for the patient, (2) in a structured experimental setting, implant the needle arrangement in a tissue phantom using the robot, and (3) compute a dose plan from the implanted needle arrangement that meets treatment dose objectives. We will confirm the needle placement accuracy and the quality of dose plans using established clinical metrics.

For this environment, the patient was modeled by a tissue phantom which included realistic representations (i.e. shapes and sizes) of the prostate, urethra, bladder, penile bulb, pubic arch, and rectum. We assumed that the tissue phantom was an 

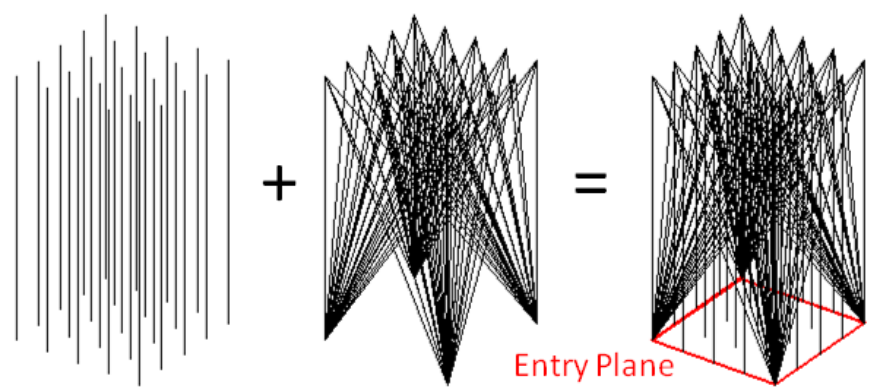

Fig. 3. The candidate needle set was the set of needles that were available during needle planning. For this study, the candidate needle set consisted of parallel lines and skew-lines (non-parallel, non-intersecting) minus any needle that intersected a non-prostate organ. The entry plane, which represents the region of the perineum where needles can enter the phantom is also shown.

acceptable model of the human prostate and nearby structures. Additionally the gelatin-based phantom was assumed to mimic human tissue stiffness properties. We also assumed that the anatomy remained unperturbed between scanning (anatomy digitization), and needle insertion. For this study we did not account for anatomy deformations during needle insertion, i.e. we assumed that needle insertions did not significantly alter the anatomy models.

A CT marker, a small radio-fluorescent pellet visible on CT-scan, was placed on the entry surface of the phantom. The CT marker was used to register the coordinate system of the planning algorithms with the robot space. We also defined an entry plane on the surface of the phantom for needles to enter. The entry plane was centered on the CT marker. We assumed that this entry plane defined the space where needles could be inserted into the perineum, which is the region where needles are inserted in an actual HDR-BT procedure. The CT marker and entry plane location is shown on a phantom in Figure 2 (left).

\section{Planning Skew-Line Needle Arrangements and DOSE DisTRIBUTIONS}

\section{A. Dose Distribution Optimization}

Dosimetric indices are quantitative metrics for dose plans. A dosimetric index, $\mathrm{V}_{d}^{\mathrm{s}}$, is the volume of organ $s$ receiving at least $d$ dose. Usually $d$ is given as a percent of the prescription dose - the dose which should be delivered to as much of the prostate as possible. $\mathrm{V}^{\text {Prostate }}$ is given as a percentage of the prostate volume, and the dosimetric indices for other organs are given in $\mathrm{cm}^{3}$. The RTOG-0321 clinical protocol [35] has established restrictions on a standard set of dosimetric indices that are correlated with positive post-treatment outcomes (i.e. high cure rates for the cancer with low rates of side effects). These restrictions are summarized in the second column of Table I.

Dose plans were optimized to a given needle arrangement using Inverse Planning by Integer Program (IPIP), a dose planning optimization model that directly optimizes dosimetric indices. The IPIP model and optimization parameters were the same as in [22]. A prescription dose of $950 \mathrm{cGy}$ was applied to all planning cases, which is a common prescription dose for prostate HDR-BT.

\section{B. Skew-Line Needle Arrangements Optimization}

There are currently no standard metrics for the quality of an HDR-BT needle arrangement. However, three properties are desired for needle arrangement: (1) it should be able to produce a dose plan that meets all dose objectives, (2) it should use needles economically, and (3) it should not contain needles which penetrate non-prostate organs. Despite the desire to avoid puncturing, sometimes organs such as the penile bulb are punctured in practice. Therefore, we propose the organ volume displaced by needles as a "trauma metric" for needle arrangements. Specifically the trauma metric for organ $s$ induced by a needle arrangement is

$$
T^{s}=\sum_{k} A_{k} L_{k}^{s}
$$

where $A_{k}$ is the cross sectional area of needle $k$ and $L_{k}^{s}$ is the length of needle $k$ puncturing organ $s$.

Needle arrangements were optimized using Needle Planning by Integer Program (NPIP) [34]. A candidate needle set was generated for the patient. This candidate needle set consisted of parallel lines and skew-lines. Skew-lines are non-parallel, non-intersecting lines. Although the needles intersected at four base points in the non-parallel set, these points were outside the prostate. Inside the prostate, the lines were nonintersecting. The candidate needle set was chosen to (1) be within the workspace of the robot, (2) cover the space of needle arrangements possible with the degrees of freedom of the robot, and (3) contain mostly needles that did not intersect the penile bulb. A picture of the candidate needle set is shown in Figure 3. Needles that intersected organs other than the prostate were removed from the set, making the theoretical trauma metric for any non-prostate organ zero. Note that organs may still be punctured in practice due to needle placement inaccuracy.

The target volume was discretized into a uniform grid of voxels. For a user specified parameter, $\delta$, NPIP chose the smallest subset of the candidate needle set for implantation such that every voxel was within $\delta$ of at least one dwell position in the chosen set.

\section{ACUBOT-RND}

This study used the Acubot-RND system developed at Johns Hopkins University [10]. Custom software was written for the Acubot-RND to automate needle insertion. The hardware and software components are described in detail in the following paragraphs.

Hardware Specifications

Acubot-RND is a 7-DoF robot designed specifically for needle insertion. The $7 \mathrm{DoF}$ are separated into three components of Acubot-RND: a 3-DoF Cartesian Positioning Stage (CPS), a 2-DoF Rotating Center of Motion (RCM), and a 2-DoF Rotating Needle Driver Module (RND). Figure 1 shows the degrees of freedom of the AcubotRND. The RND is supported by the RCM, which sits on a passive positioning arm. The arm can be manually adjusted using tightening screws to position the RCM in a default 
configuration. The positioning arm is unactuated and rests on the CPS.

The CPS can move in the $200 \mathrm{~mm}$ along the x-axis, 50 $\mathrm{mm}$ along the $\mathrm{y}$-axis, and $50 \mathrm{~mm}$ along the $\mathrm{z}$-axis. The CPS has a spatial resolution of $0.01 \mathrm{~mm}$ [26]. A trapezoid velocity profile was used for the CPS (Velocity: $10 \mathrm{~mm} / \mathrm{s}$, Acceleration: $2 \mathrm{~mm} / \mathrm{s}^{2}$ ).

The RCM is a robotic orientation module that aligns the RND about two orthogonal axes while maintaining a fixed location for the tip of the outer nozzle of the needle holder (i.e. the center of motion). The inverse kinematics of the RCM joints to hold the needle tip in place while orienting it are built into the original Acubot-RND software. The default RCM joints have ball-worm motors which do not have rotation hard stop limits. Hence the RCM did not have a built-in homing sequence to return it to a fixed orientation in space. Therefore, the Acubot-RND was operated under manual joystick control. The system was modified to allow for a software homing procedure which is essential for autonomous operation of the robot. The range of the RCM joints were set to allow $150^{\circ}$ of rotation $\left(-75^{\circ}\right.$ to $\left.75^{\circ}\right)$ in both axes with a resolution of $0.01^{\circ}$. A trapezoid velocity profile was also used for the RCM (Velocity: $8 \%$, Acceleration: $2 \% \mathrm{~s}^{2}$ ).

The RND can insert and rotate the needle. The RND performs insertion to a maximum depth of $120 \mathrm{~mm}$ using a trapezoid velocity profile with maximum velocity if $10 \mathrm{~mm} / \mathrm{s}$ and acceleration of $2 \mathrm{~mm} / \mathrm{s}^{2}$. The needle insertion DoF has a spatial resolution of $0.01 \mathrm{~mm}$. Needle rotation was not used in this experiment. However, it has been shown that needle rotation improves targeting accuracy [33]. The RND has a needle release mechanism which enables the user to command it to release the needle at anytime during the procedure. The RND also is equipped with force sensors at the needle-guide. These sensors were not used in this study, but could be used in future work to improve placement precision.

In Figure 1 (left), DoF 1-3 belong to the CPS, DoF 4-5 belong to the RCM, and DoF 6-7 belong to the RND.

Software Specifications Acubot-RND by design operates under manual joy-stick control to perform needle insertion. Specifically, each joint can be individually selected and controlled by an operator using a joy-stick interface. Autonomous operation was not previously possible because the unactuated positioning arm and the lack of stop limits on the RCM ball-worm motors did not allow a fixed reference space for the RCM. The original control system for Acubot-RND was augmented with custom software to allow automated needle insertion using an assumed hardware configuration, i.e. default orientation for each DoF and positioning arm orientation. The system takes as input a pair of cartesian coordinates: the insertion point of the needle on the entry plane and the target point inside the phantom. The input is passed from planner to the Acubot-RND as a formatted text file of coordinate pairs, one pair for each needle.

For inverse kinematic calculations, a specific orientation of the hardware was assumed. For this study, the RCM was oriented using the positioning arm such that 0 degrees in both RCM DoF resulted in the RND oriented along the z-positive axis. Furthermore, the rotation of one RCM joint resulted in a rotation of the RND end effector in only the x-z plane, $\Theta_{x z}$, and the other RCM joint swept only in the y-z plane, $\Theta_{y z}$. This orientation had the $\mathrm{x}$-axis and $\mathrm{z}$-axis of the robot space perpendicular to gravity. The Acubot-RND was brought into this orientation by carefully fixing the positioning arm using a level.

Let $P_{0}=\left(x_{0}, y_{0}, z_{0}\right)$ and $P_{f}=\left(x_{f}, y_{f}, z_{f}\right)$ denote a cartesian coordinate input pair for our custom software. To implant the needle represented by this pair, a mapping of the $3 \mathrm{D}$ coordinate space to the $6 \mathrm{D}$ joint space of the AcubotRND must be calculated. To simplify inverse kinematics, the insertion of the needle specified by these points was decoupled into three sequential tasks. (1) The tip of the needle was moved to $P_{0}$ using only the actuation in the CPS. (2) The RCM joints were actuated to orient the RND toward $P_{f}$. According to the physical orientation of the Acubot-RND used for this study, the angles of the two RCM joints were

$$
\Theta_{x z}=\tan ^{-1}\left(\frac{x_{f}-x_{0}}{z_{f}-z_{0}}\right) \text {, and } \Theta_{y z}=\tan ^{-1}\left(\frac{y_{f}-y_{0}}{z_{f}-z_{0}}\right) \text {. }
$$

(3) The needle tip was inserted to $P_{f}$ by actuating the needle insertion DoF of the RND a depth of $d=\left\|P_{f}-P_{0}\right\|$.

After the needle was inserted to the target point, the robot was retracted back to the insertion point $\left(P_{0}\right)$ using the RND, and the RCM was then re-orientated to zero position.

The software was also set to pause the needle insertion operation at critical points in the execution and wait for an operator command to continue. These stopping points were between needle insertions, before releasing the needle from the RND (discussed in Section VI), and orienting the RCM back to zero position after needle insertion.

Integration with Needle Planning The entry plane is defined as the planar segment where needles can be inserted. The size of the entry plane is limited by the maximum ranges of the $\mathrm{x}$ - and $\mathrm{y}$-DoF of the CPS because the needle tip is brought to the needle insertion point on the entry plane using only these joints. The maximum ranges of these joints result in a rectangle $200 \mathrm{~mm}$ in the $\mathrm{x}$-direction and $50 \mathrm{~mm}$ in the $y$-direction. In case of calibration or other setup errors, a conservative entry plane of $45 \mathrm{~mm} \times 45 \mathrm{~mm}$ was used for this study. This entry plane is smaller than the standard template for PPI- and HDR-BT, which is $100 \mathrm{~mm} \times 100 \mathrm{~mm}$. This entry plane is shown on the phantom in Figure 2 and relative to the candidate needle set in Figure 3.

\section{System Design AND ARChitecture}

The robot implant workflow consisted of pre-implant scanning and planning, robot setup and calibration, needle implanting, and post-implant scanning and dose planning. Each step is discussed in detail in the following paragraphs.

Pre-Implant Scanning and Planning A CT scan was performed on each phantom. The organs of the phantom 
and the CT marker were segmented using the Nucletron Oncentra ${ }^{\circledR}$ Dynamic Planning Environment. The 3D organ models were exported to NPIP. A candidate needle set was generated, and NPIP computed a needle arrangement for each phantom. A dose plan was also generated using IPIP to verify that the planned needle arrangement could produce a clinically acceptable dose plan. The needle insertion and target points for each needle arrangement were written to a formatted text file for our custom software.

Robot Setup and Calibration The phantom was moved to the robot work area and clamped to the table to ensure immobility during the procedure. The list of cartesian coordinate pairs specifying the needle arrangement was input to Acubot-RND.

The robot was calibrated to the position of the phantom by simultaneously (1) putting the robot in zero position, (2) placing the needle tip on the CT marker, and (3) placing the phantom such that the translational axis, $x-D o F$ and $y-D o F$, of the CPS were parallel to the entry surface of the phantom. Parallelism of the X-Y plane of the robot space to the entry surface was determined by moving the needle tip in the $\mathrm{x}$ axis and y-axis of CPS using the joy-stick controller. A visual inspection was made to determine if the needle tip was always the same distance from the phantom surface. We refer to this calibrated positioning of robot and phantom as the initial state. Figure 2 shows the Acubot-RND and phantom in initial state.

The Acubot-RND used an 18-gauge, $15 \mathrm{~cm}$ long, diamondtip brachytherapy needle (COOK Biotech) for this procedure. The needle is a $2 \mathrm{~mm}$ diameter hollow sheath which houses a rigid stylet. Since Acubot-RND was not designed for multiple needle insertions in HDR-BT, needle segments were cut from the stylets of regular brachytherapy needles. These needle segments were used to represent the hollow needles that would be inserted in a regular HDR-BT procedure and connected to an afterloader for dose delivery.

Needle Implant Workflow To implant the input needle arrangement, the Acubot-RND was brought into initial state, and each needle was inserted sequentially according to the following procedure:

(1) Acubot-RND reached an insertion point in zero position.

(2) Acubot-RND aligned needle towards the target point.

(3) Acubot-RND inserted the needle tip to the target point.

(4) Acubot-RND halted for needle segment insertion.

(5) Operator removed the Acubot-RND stylet.

(6) Operator insertsed a needle segment to needle tip.

(7) Operator manually held the stylet.

(8) Acubot-RND released the needle.

(9) Operator pulled the needle over the stylet.

(10) Needle segment was left in place.

(11) Acubot-RND returned to zero position.

(12) Operator placed needle and stylet back into RND.

Note that the Steps 1, 8, and 11 were initiated on operator command.

Post-Implant-Scanning and Dose Planning

After exe-

\begin{tabular}{|l|l|l|l|l|l|l|}
\hline & & \multicolumn{2}{|c|}{ Phantom 1 } & \multicolumn{2}{l|}{ Phantom 2 } & Human \\
\hline Index & Req. & P1 & A1 & P2 & A2 & \\
\hline \hline $\mathrm{V}_{100}^{\text {Prostate }}$ & $\geq 90 \%$ & 99.0 & 97.0 & 96.0 & 96.0 & 98.0 \\
\hline $\mathrm{V}_{150}^{\text {Prostate }}$ & $\leq 45 \%$ & 39.0 & 40.0 & 40.0 & 37.0 & 37.0 \\
\hline $\mathrm{V}_{75}^{\text {Bladder }}$ & $\leq 1 \mathrm{~cm}^{3}$ & 0.00 & 0.00 & 0.30 & 0.80 & 0.30 \\
\hline $\mathrm{V}_{100}^{\text {Bladder }}$ & $=0 \mathrm{~cm}^{3}$ & 0.00 & 0.00 & 0.00 & 0.00 & 0.00 \\
\hline $\mathrm{V}_{75}^{\text {Bulb }}$ & $\leq 1 \mathrm{~cm}^{3}$ & 0.00 & 0.00 & 0.00 & 0.00 & 0.00 \\
\hline $\mathrm{V}_{100}^{\text {Bulb }}$ & $=0 \mathrm{~cm}^{3}$ & 0.00 & 0.00 & 0.00 & 0.00 & 0.00 \\
\hline $\mathrm{V}_{75}^{\text {Rectum }}$ & $\leq 1 \mathrm{~cm}^{3}$ & 0.06 & 0.00 & 0.00 & 0.00 & 0.00 \\
\hline $\mathrm{V}_{100}^{\text {Rectum }}$ & $=0 \mathrm{~cm}^{3}$ & 0.00 & 0.00 & 0.00 & 0.00 & 0.00 \\
\hline $\mathrm{V}_{125}^{\text {Urethra }}$ & $\leq 1 \mathrm{~cm}^{3}$ & 0.06 & 0.05 & 0.04 & 0.06 & 0.07 \\
\hline $\mathrm{V}_{150}^{\text {Urethra }}$ & $=0 \mathrm{~cm}^{3}$ & 0.00 & 0.00 & 0.00 & 0.00 & 0.00 \\
\hline $\mathrm{V}_{100}^{\text {Body }}$ & $=0 \mathrm{~cm}^{3}$ & 0.00 & 0.00 & 0.00 & 0.00 & 0.00 \\
\hline $\mathrm{T}^{\text {Bulb }}$ & min & 0.00 & 0.00 & 0.00 & 0.00 & 0.00 \\
\hline
\end{tabular}

TABLE I

DOSE AND TRAUMA METRICS FOR IMPLANTS BY ROBOTIC SYSTEM (PLANNED (P) AND ACTUAL (A)) AND FOR IMPLANT BY EXPERT

cuting the implant, a second CT scan was obtained of the phantom. The metal needle segments could be identified but created significant artifacts on the CT scan. This made segmenting the anatomy rather difficult. However, since the phantom was static and provided many rigid control points, co-registration between the pre-implant and post-implant CT scans could be performed without introducing significant uncertainty. Coregistration is a standard feature in the Oncentra planning system. The needle positions and co-registered anatomy were used to compute a second dose plan using IPIP. Relevant dosimetric indices, trauma metrics, and error measurements were computed and are listed in I.

\section{EXPERT (HUMAN PHYSICIAN) IMPLANTS}

To compare our system with the performance of a physician on a similar task, an implant was performed on a tissue phantom by a Dr. I-Chow Hsu (the fifth author), a professor of radiation oncology at UCSF (expert). The expert is a certified radiation oncologist with a specialization in brachytherapy and over 18 years of clinical experience. The expert inserted 16 standard HDR-BT needles into the phantom under transrectal ultrasound (TRUS) guidance using the UCSF-developed freehand technique [36]. A HAWK 2102 EXL TRUS system from B-K Medical was used for ultrasound imaging. Postimplant scanning and dose planning was performed using the same method as for the robot implants.

\section{RESULTS}

There were three custom tissue phantoms constructed for this study: two for tests with the robot and one for the expert. There were two needle arrangements defined for each phantom: the pre-implant NPIP arrangement (planned) and the post-implant arrangement (actual). We label the needle arrangements computed by NPIP for the two phantoms as P1 and $\mathrm{P} 2$ to distinguish them from the actual implanted needle arrangements, A1 and A2. Since the expert implant was done 


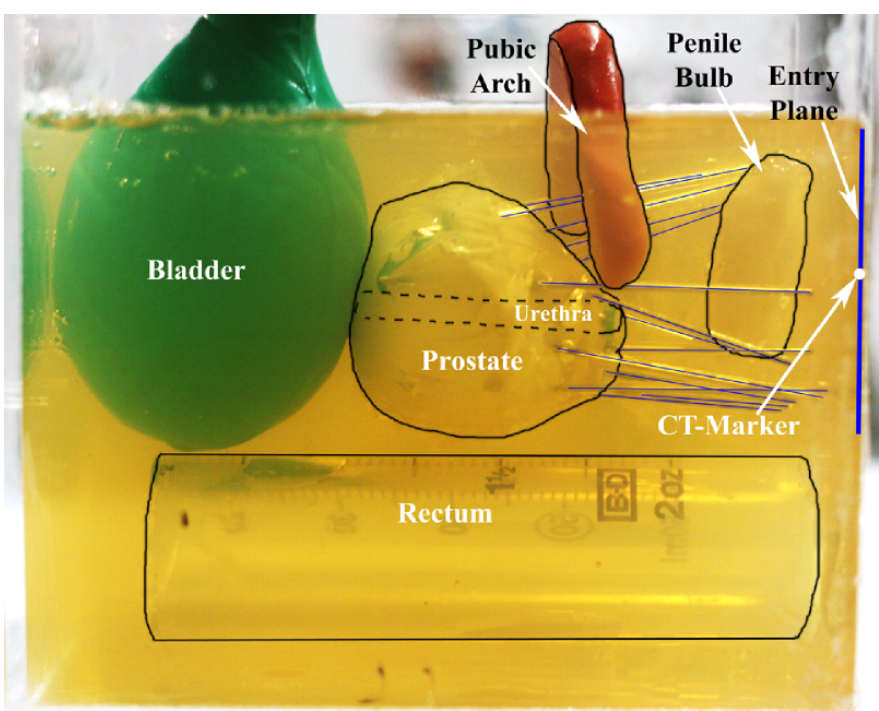

Fig. 4. Photograph of tissue phantom with robot-implanted needle segments. The organ boundaries and needle patterns are superimposed on the figure for visual aid. No non-prostate organ was punctured in the insertion of this implant.

manually, there was no pre-implant needle plan. We label the third phantom implanted by the expert as Human.

The CT scans of the tissue phantoms were taken in 3 $\mathrm{mm}$ thick slices. The contoured prostate volumes for the three phantoms were $39 \mathrm{~cm}^{3}, 32 \mathrm{~cm}^{3}$, and $37 \mathrm{~cm}^{3}$. The total phantom volume was $750 \mathrm{~cm}^{3}$. The regions of interest were contoured using Oncentra $^{\circledR}$ version 4.1 by a medical physicist. Note that a clinician in radiotherapy, even one without brachytherapy experience, would have basic proficiency in organ contouring. Therefore, although the novice did not perform the contouring in this study, it can be assumed that a novice clinician using our robotic system would be able to do it. There was a $2 \mathrm{~mm}$ margin added to the contours of the penile bulb to ensure small placement errors did not result in puncturing of the bulb. Specifically, the contours of the bulb were made $2 \mathrm{~mm}$ larger in every direction. Adding margins is a common tool in radiation oncology dose planning and is a standard feature in Oncentra.

The number of needles in the NPIP candidate needle set after needles intersecting non-prostate organs were removed was 287 and 229 for P1 and P2, respectively. The NPIP $\delta$ parameter [34] was fine-tuned until NPIP returned a needle arrangement with 16 needles, the standard number used for HDR-BT at the UCSF clinic. This corresponded to a $\delta$ value of $6.5 \mathrm{~mm}$ for the first phantom and $6.0 \mathrm{~mm}$ for the second phantom. All computational tasks were performed using Matlab R2011a on a Lenovo ThinkPad with an Intel i5-2410M processor and 4GB of RAM. The integer program optimization was done using the Matlab interface for the Mosek Optimization Toolbox v.6 [37].

The computed needle arrangements were implanted into each phantom in approximately 45 minutes plus setup time. A side view of an implanted phantom is shown in Figure 4. The expert implant took approximately 15 minutes to execute.

Relevant dosimetric indices and trauma metrics are shown in Table I. Note that the quality of a dose distribution is only related to compliance with the dose criteria, not by the level of compliance. In other words, there is no clinical evidence to show that $\mathrm{V}_{100}^{\text {Prostate }}=98 \%$ is better than $\mathrm{V}_{100}^{\text {Prostate }}=97 \%$ in improving long term post-treatment outcomes, only that meeting $\mathrm{V}_{100}^{\text {Prostate }} \geq 90 \%$ is better than failing this criterion.

There was a small difference between the dose plan from the planned arrangements and the dose plan from the actual arrangements for most of the indices. The one exception is the difference in $\mathrm{V}_{75}^{\text {Bladder }}$ values for $\mathrm{P} 2$ and $\mathrm{A} 2$ which were $0.3 \mathrm{~cm}^{3}$ and $0.8 \mathrm{~cm}^{3}$, respectively. The clinically acceptable limit for this criterion is $1 \mathrm{~cm}^{3}$. This discrepancy is due to the needles not being inserted far enough into the prostate. As a result, the dwell times at the superior section of the prostate were increased by the dose planning system to cover more of the prostate with the prescription dose. The increased dwell times at the apex of the prostate resulted in slightly higher bladder dose because the bladder is above the prostate.

The trauma metric computed for this study was $\mathrm{T}^{\text {bulb }}$ because it was the only organ that was at risk of being punctured in our setup. The trauma metric was computed using $A_{k}=\pi r^{2} \mathrm{~mm}^{2}$ for all $k$ using $r=1 \mathrm{~mm}$ (standard HDRBT needles have $1 \mathrm{~mm}$ radius) and $L_{k}^{\text {bulb }}$ was computed by counting the number of contour slices the needle was within the bulb and multiplying it by the CT-Scan slice thickness, which was $3 \mathrm{~mm}$. The bulb contours without the $2 \mathrm{~mm}$ margins were used to compute this metric. However, the trauma metric for the bulb was $0.00 \mathrm{~mm}^{3}$ for every case because the bulb was not punctured. However, with a different experimental setup or physician, this may not be the case.

A superposition of the planned (red) and actual (blue) needle arrangement is shown in Figure 5 for both phantoms. An offset error was computed as the rigid displacement of the actual needle arrangement that achieved the least squared error between the tips of the needles in the planned and actual arrangement. For this calculation, the tips were taken to be the first and last dwell positions within the prostate. This is a fair approximation of the needle segments because the needles were rigid and straight. Between P1 and A1, there was an offset error of $-1.6 \mathrm{~mm}$ in the $\mathrm{x}$-direction, $-1.7 \mathrm{~mm}$ in the $\mathrm{y}$ direction, and $-0.9 \mathrm{~mm}$ in the $\mathrm{z}$-direction. The mean distance between needle tips was $3.1 \mathrm{~mm}$. Between P2 and A2, there was an offset error of $-0.8 \mathrm{~mm}$ in the $\mathrm{x}$-direction, $-2.2 \mathrm{~mm}$ in the y-direction, and $-1.8 \mathrm{~mm}$ in the z-direction. The mean distance between needle tips was $4 \mathrm{~mm}$. Accounting for offset correction in the actual needle arrangement, the mean distance between needle tips was $1.8 \mathrm{~mm}$ for P1-A1, and $2.8 \mathrm{~mm}$ for P2A2.

\section{Discussion}

These experiments have confirmed that computationally generated needle arrangements can be planned and executed using our robotic system with sufficient accuracy to achieve treatment objectives in our experimental setup.

Our system had non-trivial placement errors which can be attributed to the following two sources: (1) error in registration 

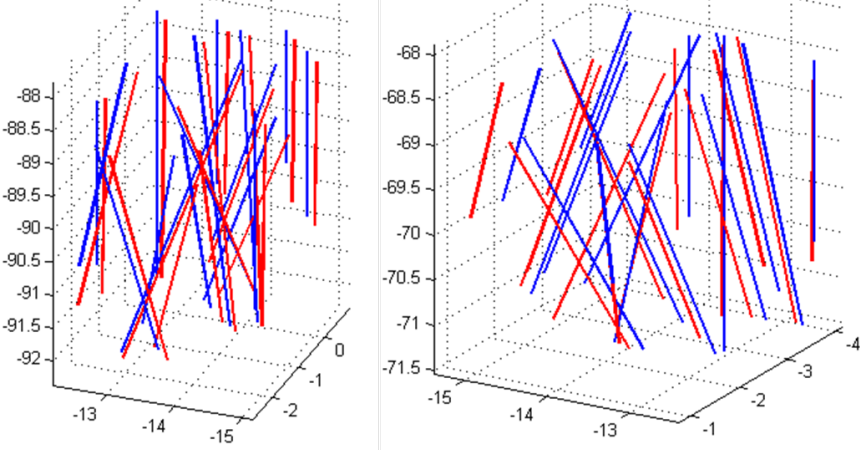

Fig. 5. Superposition of planned (red) and actual (blue) needle arrangement between P1 and A1 (left) and P2 and A2 (right). Although no sensitive structure was punctured in the actual needle arrangement and all dose objectives were met, there was placement error due to calibration offset and operator error.

to the phantom, (2) error in co-registration of the CT images, and (3) operator error during needle retraction (i.e. the novice did not hold the needle exactly in place when retracting the needle over the stylet). Registration seemed to be the largest source of error since the entire needle arrangement was offset by $-1.6 \mathrm{~mm}$ in the $\mathrm{x}$-direction, $1.7 \mathrm{~mm}$ in the $\mathrm{y}$-direction, and $-0.9 \mathrm{~mm}$ in the z-direction for the first phantom and $-0.8 \mathrm{~mm}$ in the $\mathrm{x}$-direction, $-2.2 \mathrm{~mm}$ in the $\mathrm{y}$-direction, and $-1.8 \mathrm{~mm}$ in the z-direction for the second phantom. Operator error is most likely to manifest along the direction of needle insertion, as in A2 where the needles were not inserted far enough. Registration error may be reduced by improving the calibration of the robot to the phantom with a custom fixture, which we will explore in future work. Additionally, using force feedback and real-time image registration to make this system closedloop can assist in realizing the planned needle arrangement. A novice operator was required to insert the needle segment into the phantom because Acubot-RND hardware does not support automatic needle segment dropping. With specialized hardware adjustment in Acubo-RND, the dependency on novice can be reduced leading to lower placement error. According to other studies with PPI-BT robots, sub-millimeter precision is possible $[10,32,33]$.

The ability to execute computationally generated needle arrangements that avoid puncturing critical structures has the potential to significantly reduce side effects while still accomplishing treatment objectives. Figure 6 shows a crosssection of the needle arrangement implanted by the expert (left) and by the robot (right). It is evident from the figure that the needles in the expert implant are distributed evenly around the periphery and inside of the prostate. The robot implant is crisscrossed in the prostate and there is a deficit of needles in the posterior of the prostate. Absence of needle planning in expert implant results in insertions which are evenly distributed. While our systems pre-computes a needle arrangement for a particular anatomy which might not be symmetric. Despite the shortcomings in accuracy of the robotic needle implant, it should be considered a major step because it required little operator skill and all clinical treatment
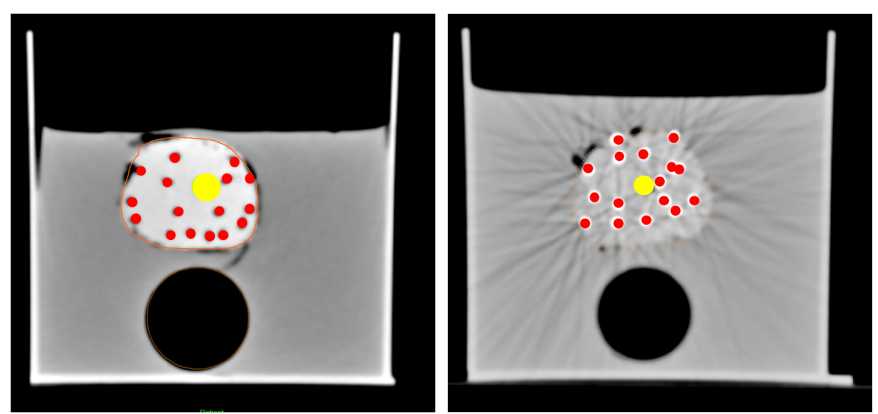

Fig. 6. CT slice of physician implant (left) and robot implant (right). The expert implant has needles evenly distributed around the periphery and inside of the prostate. While the robot implants needles which are criss-crossed. Our semi-autonomous system pre-computes optimal anatomy specific needle arrangements which might not be symmetrical but are able to meet treatment objectives and reduce trauma to sensitive organs at risk. Future improvements to needle insertion hardware and planning software will result in improved treatment quality, reproducibility and repeatability.

objectives were achieved. We conclude that planning software and robot hardware tools exist to perform robotic HDR-BT and that the development of specialized robotic hardware for HDR-BT has the potential to reduce side effects and increase the accessibility of treatment.

\section{CONCLusion}

We describe the system architecture, algorithms, hardware, interface, and experiments demonstrating the first semiautomated robot implementation of skew-line needle arrangements for HDR-BT. Results suggest that the resulting skewline needle arrangements are comparable to those achieved by an expert human physician; both meet clinical objectives and avoid sensitive organ structures. In future work, we will add fixtures to improve calibration and refine the planning algorithm to anticipate and compensate for tissue deformation.

\section{ACKNOWLEDGMENT}

This research was funded in part by NIH-1R01EB-00643501A1, NSF-0905344, and American Cancer Society Grant \#IRG-97-150-13. The authors thank the staff at UCSF Medical Center at Mt. Zion for their assistance with the physician and robotic implants and for generously providing access to their research labs, clinical supplies, CT-scanner, and Nucletron Oncentra ${ }^{\circledR D}$ ynamic Planning Environment. The authors also thank Neil Zhang for constructing the phantoms, Yihe Zuo for assistance in troubleshooting the Acubot-RND, and Heimdall Siao for helping with software components of Acubot-RND.

\section{REFERENCES}

[1] K. Wallner, J. Blasko, and M. Datolli, Prostate Brachytherapy Made Complicated. Seattle, WA: Smart Medicine Press, 2001.

[2] S. K. Kang, R. H. Chou, R. K. Dodge, R. W. Clough, H.-S. L. Kang, M. G. Bowen, B. A. Steffey, S. K. Das, S.-M. Zhou, A. W. Whitehurst, N. J. Buckley, J. H. Kim, R. E. Joyner, I. Sarmina, G. S. Montana, S. S. Ingram, and M. S. Anscher, "Acute urinary toxicity following transperineal prostate brachytherapy using a modified Quimby loading method," Int. J. Radiat. Oncol., Biol., Phys., vol. 50, no. 4, pp. 937-945, 2001.

[3] L. Eapen, C. Kayser, Y. Deshaies, G. Perry, C. E, C. Morash, J. E. Cygler, D. Wilkins, and S. Dahrouge, "Correlating the degree of needle trauma during prostate brachytherapy and the development of acute urinary toxicity," Int. J. Radiat. Oncol., Biol., Phys., vol. 59, no. 5, pp. 1392-1394, 2004 
[4] C. Vargas, M. Ghilezan, M. Hollander, G. Gustafson, H. Korman, J. Gonzalez, and A. Martinez, "A new model using number of needles and androgen deprivation to predict chronic urinary toxicity for high or low dose rate prostate brachytherapy," J. Urology, vol. 174, no. 3, pp. 882-887, September 2005.

[5] R. M. Munarriz, Q. R. Yan, A. Nehra, D. Udelson, and I. Goldstein, "Blunt trauma: The pathophysiology of hemodynamic injury leading to erectile dysfunction," J. Urology, vol. 153, no. 6, pp. 1831-1840, June 1995.

[6] B. M. Fisch, B. Pickett, V. Weinberg, and M. R. III, "Dose of radiation received by the bulb of the penis correlates with risk of impotence after three-dimensional conformal radiotherapy for prostate cancer," Urology, vol. 57, no. 5, pp. 955-959, May 2001.

[7] G. S. Merrick, K. Wallner, W. M. Butler, R. W. Galbreath, J. H. Lief, and M. L. Benson, "A comparison of radiation dose to the bulb of the penis in men with and without prostate brachytherapy-induced erectile dysfunction," Int. J. Radiat. Oncol., Biol., Phys., vol. 50, no. 3, pp. 597-604, July 2001

[8] J. A. M. Cunha, I.-C. Hsu, and J. Pouliot, "Dosimetric equivalence of nonstandard HDR brachytherapy catheter patterns," Medical Physics, vol. 36, no. 1, pp. 233-239, January 2009.

[9] Y. Kim, I.-C. J. Hsu, and J. Pouliot, "Measurement of craniocaudal catheter displacement between fractions in computed tomography-based high dose rate brachytherapy of prostate cancer," J Appl Clin Med Phys, vol. 8, no. 4, pp. 1-13, 2007.

[10] D. Stoianovici, K. Cleary, A. Patriciu, D. Mazilu, A. Stanimir, N. Craciunoiu, V. Watson, and L. Kavoussi, "Acubot: a robot for radiological interventions," Robotics and Automation, IEEE Transactions on, vol. 19, no. 5, pp. 927 - 930, oct. 2003.

[11] Y. Huang and K. Low, "Comprehensive planning of robotic therapy and assessment of task-oriented functions via improved qfd applicable to hand rehabilitation," in Automation Science and Engineering (CASE), 2010 IEEE Conference on. IEEE, 2010, pp. 252-257.

[12] J. Wang and Y. Li, "Hybrid impedance control of a 3-dof robotic arm used for rehabilitation treatment," in Automation Science and Engineering (CASE), 2010 IEEE Conference on. IEEE, 2010, pp. 768773.

[13] M. Chandrapal, X. Chen, and W. Wang, "Self organizing fuzzy control of pneumatic artificial muscle for active orthotic device," in Automation Science and Engineering (CASE), 2010 IEEE Conference on, aug. 2010, pp. $632-637$.

[14] M. Ashraf, S. Tayyaba, and N. Afzulpurkar, "Mems based polymeric drug delivery system," in Automation Science and Engineering (CASE), 2010 IEEE Conference on, aug. 2010, pp. $192-197$.

[15] A. Ratnakar and M. Zhou, "An ultrasound system for tumor detection in soft tissues using low transient pulse," in Automation Science and Engineering (CASE), 2011 IEEE Conference on, aug. 2011, pp. 684 -689 .

[16] B. J. Choi, W. S. You, S. H. Shin, H. Moon, J. C. Koo, W. Chung, and H. R. Choi, "Development of robotic laboratory automation platform with intelligent mobile agents for clinical chemistry," in Automation Science and Engineering (CASE), 2011 IEEE Conference on, aug. 2011, pp. $708-713$.

[17] Y. Yu, J. B. Zhang, R. A. Brasacchio, P. G. Okunieff, D. J. Rubens, J. G. Strang, A. Soni, and E. M. Messing, "Automated treatment planning engine for prostate seed implant brachytherapy." International journal of radiation oncology, biology, physics, vol. 43, no. 3, pp. 647-52, February 1999. [Online]. Available: http://www.ncbi.nlm.nih.gov/pubmed/10078652

[18] M. Lahanas, D. Baltas, and N. Zamboglou, "Anatomy-based threedimensional dose optimization in brachytherapy using multiobjective genetic algorithms," Medical Physics, vol. 26, no. 9, pp. 1904-1918, 1999. [Online]. Available: http://link.aip.org/link/?MPH/26/1904/1

[19] E. Lessard, "Development and clinical introduction of an inverse planning dose optimization by simulated annealing "IPSA" for high dose rate brachytherapy," Medical Physics, vol. 31, p. 2935, 2004.

[20] R. Alterovitz, E. Lessard, J. Pouliot, I.-C. Hsu, J. F. O’Brian, and K. Goldberg, "Optimization of HDR brachytherapy dose distributions using linear programming with penalty costs," Medical Physics, vol. 33, no. 11, pp. 4012-4019, November 2006.

[21] A. Karabis, P. Belotti, and D. Baltas, "Optimization of Catheter Position and Dwell Time in Prostate HDR Brachytherapy using HIPO and Linear Programming," in IFMBE Proceedings, vol. 25/XI, September 2009, pp. 612-615.
[22] T. Siauw, A. Cunha, A. Atamturk, I. Hsu, J. Pouliot, and K. Goldberg, "Ipip: A new approach to inverse planning for hdr brachytherapy by directly optimizing dosimetric indices," Medical Physics, vol. 38, no. 7, pp. 4045-4051, 2011.

[23] D. P. Huyskens, P. Maingon, L. Vanuytsel, V. Remouchamps, T. Roques, B. Dubray, B. Haas, P. Kunz, T. Coradi, R. Bhlman, R. Reddick, A. V. Esch, and E. Salamon, "A qualitative and a quantitative analysis of an auto-segmentation module for prostate cancer," Radiotherapy and Oncologyl, vol. 90, no. 3, pp. 337-345, 2009.

[24] C. Schneider, A. M. Okamura, and G. Fichtinger, "A robotic system for transrectal needle insertion into the prostate with integrated ultrasound," in Proc. IEEE Int. Conf. Robotics and Automation (ICRA), May 2004, pp. 2085-2091.

[25] L. Phee, D. Xiao, J. Yuen, C. F. Chan, H. Ho, C. H. Thng, C. Cheng, and W. S. Ng, "Ultrasound guided robotic system for transperineal biopsy of the prostate," in Proc. IEEE Int. Conf. Robotics and Automation (ICRA), April 2005, pp. 1327-1332.

[26] G. Fichtinger, E. C. Burdette, A. Tanacs, A. Patriciu, D. Mazilu, L. L. Whitcomb, and D. Stoianovici, "Robotically assisted prostate brachytherapy with transrectal ultrasound guidance-phantom experiments," Brachytherapy, vol. 5, no. 1, pp. 14-26, January 2006.

[27] Y. Yu, T. K. Podder, Y. D. Zhang, W. S. Ng, V. Misic, J. Sherman, D. Fuller, D. J. Rubens, J. G. Strang, R. A. Brasacchio, and E. M. Messing, "Robotic system for prostate brachytherapy," Computer Aided Surgery, vol. 12, no. 6, pp. 366-370, 2007. [Online]. Available: http://informahealthcare.com/doi/abs/10.3109/10929080701746926

[28] A. Patriciu, D. Petrisor, M. Muntener, D. MAzilu, M. Schär, and D. Stoianovici, "Automatic brachytherapy seed placement under MRI guidance," IEEE Trans. Biomedical Engineering, vol. 54, no. 8, pp. 1499-1506, aug 2007.

[29] A. L. Trejos, R. Patel, and R. Malthaner, "A Device for Robot-Assisted Minimally-Invasive Lung Brachytherapy," in Proc. IEEE Int. Conf. on Robotics and Automation (ICRA), 2006.

[30] S. Salcudean, T. Prananta, W. Morris, and I. Spadinger, "A robotic needle guide for prostate brachytherapy," in IEEE International Conference on Robotics and Automation, may 2008, pp. 2975-2981.

[31] G. Fichtinger, J. P. Fiene, C. W. Kennedy, G. Kronreif, I. Iordachita, D. Y. Song, E. C. Burdette, and P. Kazanzides, "Robotic assistance for ultrasound-guided prostate brachytherapy," Medical Image Analysis, vol. 12 , pp. 535-545, 2008

[32] R. Pollock, P. Mozer, T. J. Guzzo, J. Marx, B. Matlaga, D. Petrisor, B. Vigaru, S. Badaan, D. Stoianovici, and M. E. Allaf, "Prospects in Percutaneous Ablative Targeting: Comparison of a Computer-Assisted Navigation System and the AcuBot Robotic System," Journal of Endourology, vol. 24, no. 8, pp. 1269-1272, August 2010.

[33] S. Badaan, D. Petrisor, C. Kim, P. Mozer, D. Mazilu, L. Gruionu, A. Patriciu, K. Cleary, and D. Stoianovici, "Does needle rotation improve lesion targeting?" The International Journal of Medical Robotics and Computer Assisted Surgery, 2011.

[34] T. Siauw, A. Cunha, A. Atamtürk, I.-C. Hsu, K. Goldberg, and J. Pouliot, "NPIP: A Computational Needle Configuration Optimization System for HDR Brachytherapy," Medical Physics, vol. Under review, 2012. [Online]. Available: http://automation.berkeley.edu/ berenson/npip.pdf

[35] I.-C. Hsu, K. Bae, K. Shinohara, J. Pouliot, J. Purdy, G. Ibbott, J. Speight, E. Vigneault, R. Ivker, and H. Sandler, "Phase II Trial of Combined High-Dose-Rate Brachytherapy and External Beam Radiotherapy for Adenocarcinoma of the Prostate: Preliminary Results of RTOG 0321," Int. J. Radiat. Oncol., Biol., Phys., vol. 78, no. 3, pp. 751-758, 2010. [Online]. Available: http://www.sciencedirect.com/science/article/pii/S0360301609030284

[36] B. Pickett and J. Pouliot, The Modern Technology of Radiation Therapy. Philadelphia: Medical Physics Publishing, 2005, vol. Vol. 2, ch. Prostate Brachytherapy.

[37] M. T. for Mosek v.6, "http://mosek.com." 